Supporting information

\title{
Exfoliated Boron Nitride (e-BN) Tailored Exfoliated Graphitic Carbon Nitride (e-CN): An Improved Visible Light Mediated Photocatalytic Approach to wards TCH Degradation and $\mathrm{H}_{2}$ Evolution
}

Lopamudra Acharya ${ }^{a}$, Sambhu Prasad Pattnaik ${ }^{a}$, Arjun Behera ${ }^{a}$, Rashmi Acharya ${ }^{a}$ and Kulamani Parida*

${ }^{a}$ Centre for Nano Science and Nano Technology, ITER, Siksha 'O' Anusandhan Deemed to be University, Bhubaneswar, Odisha, India, 751030

*Corresponding author

*Corresponding Author's Email:

kulamaniparida@soa.ac.in

paridakulamani@yahoo.com

Tel. No.: +91-674-2351777 and Fax. +91-674-2350642 

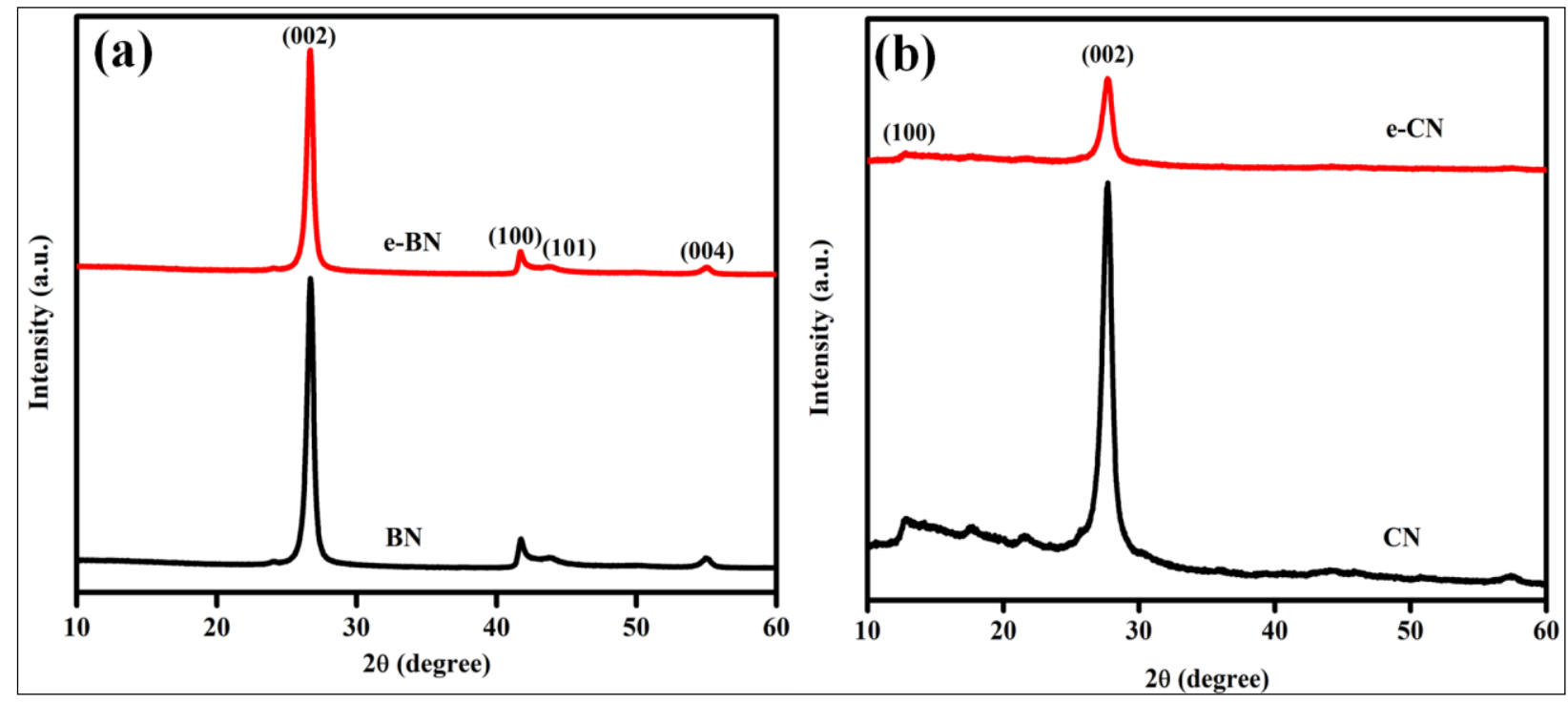

Figure S1 PXRD pattern for (a) BN and e-BN, (b) $\mathrm{CN}$ and e-CN.

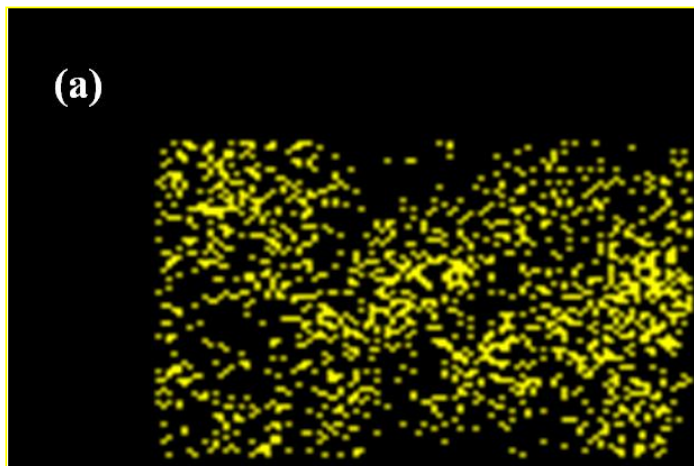

(b)

B

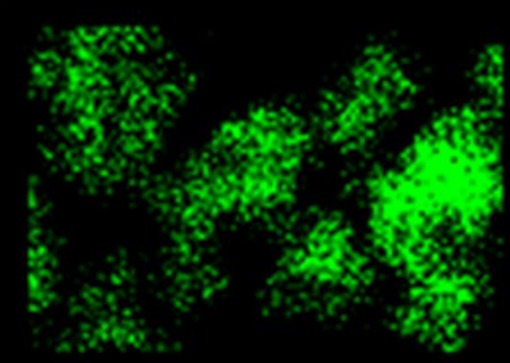

C
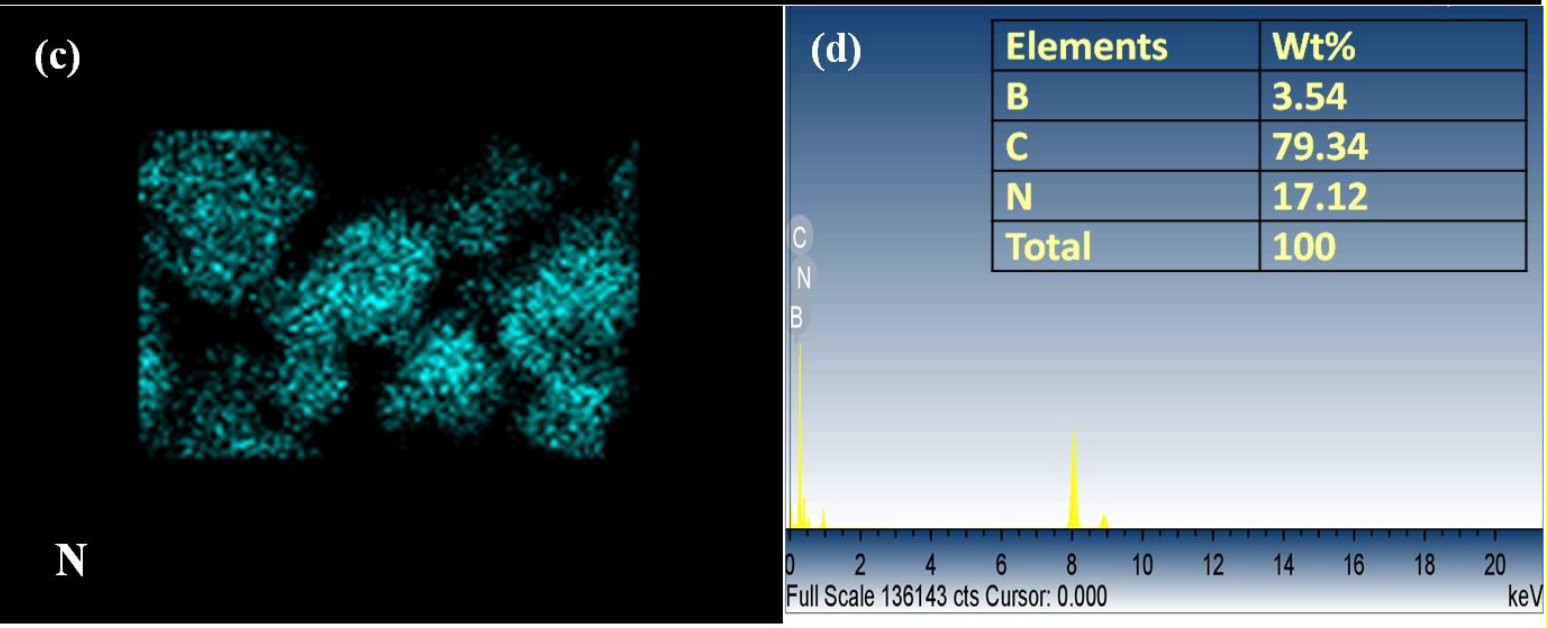
Figure S2 Elemental mapping of (a) B, (b) C and (c) N, (d) EDX spectra for $1.5 \mathrm{wt} \%$ e-BN/eCN.

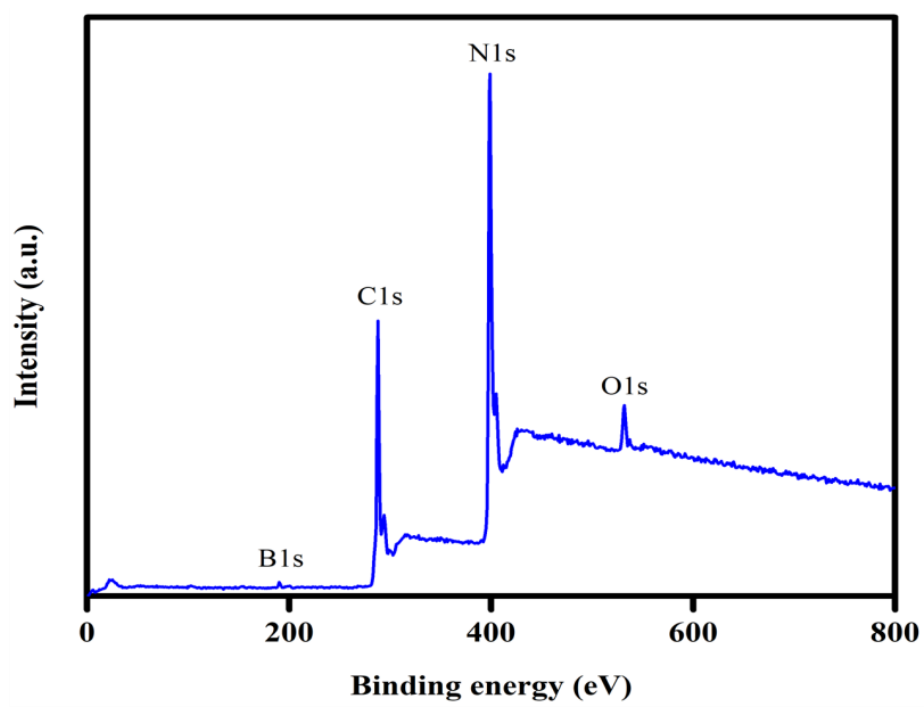

Figure S3 Survey spectra for $1.5 \mathrm{wt} \%$ e-BN/e-CN.
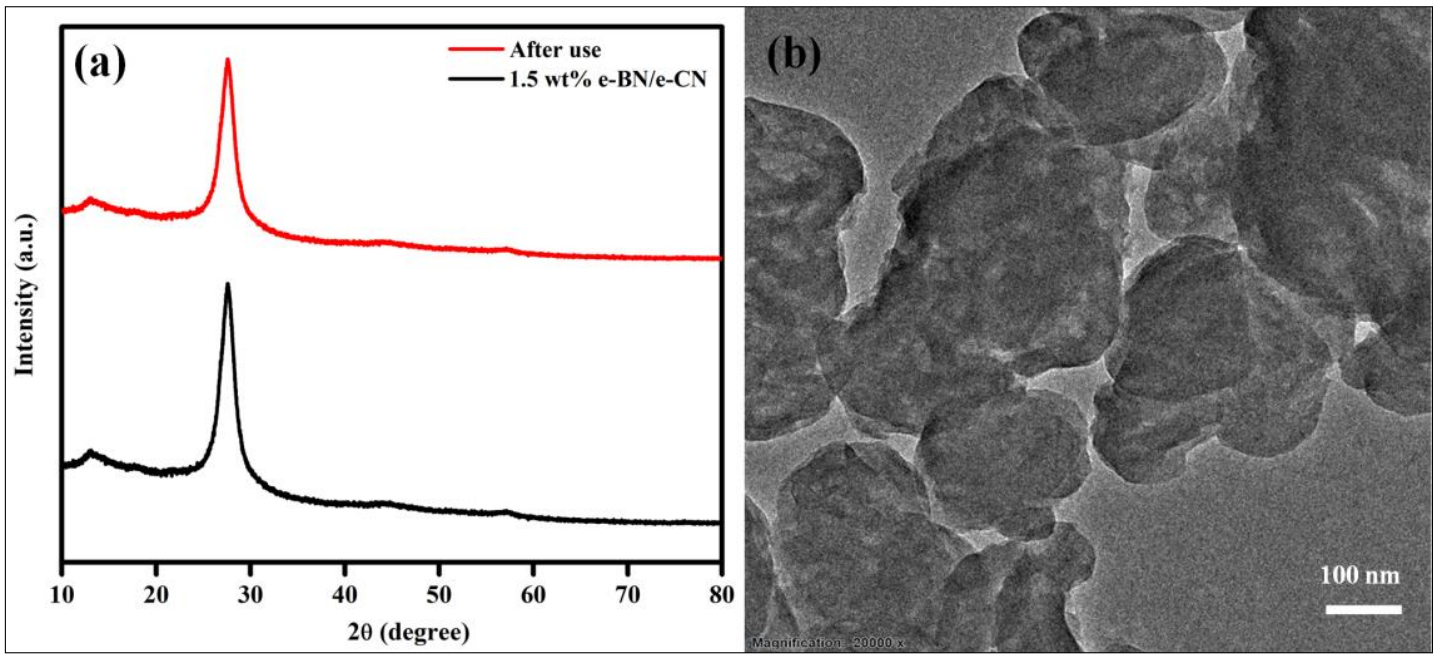

Figure S4 (a) PXRD and (b) HRTEM images of $1.5 \mathrm{wt} \%$ e-BN/e-CN photocatalyst after recycle test for $\mathrm{TCH}$ degradation and $\mathrm{H}_{2}$ evolution. 

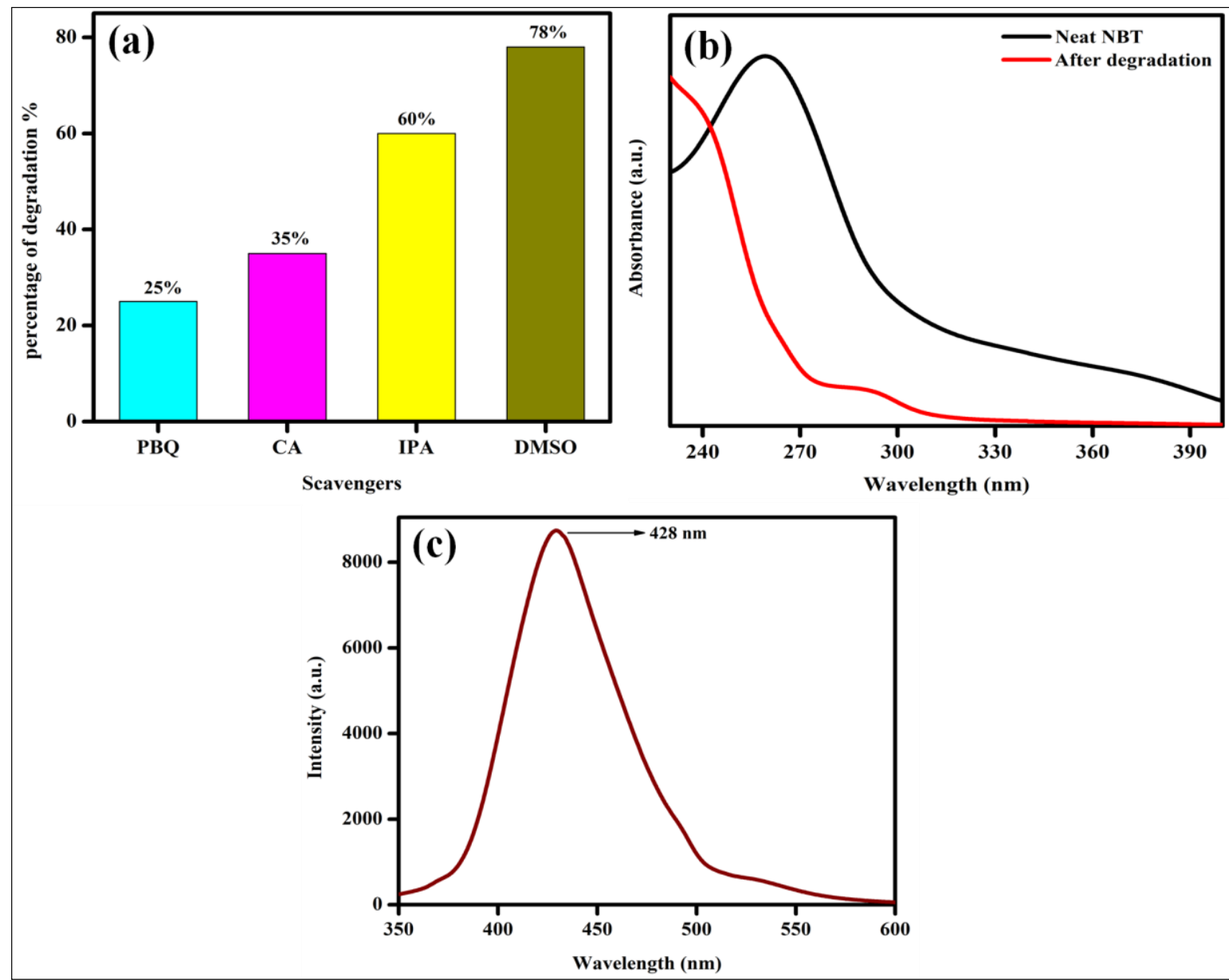

Figure S5 (a) Trapping test for $1.5 \mathrm{wt} \%$ e-BN/e-CN composite in the presence of different scavenging agents, $(b, c)$ confirmatory tests for $\bullet \mathrm{O}_{2}^{-}$and $\bullet \mathrm{OH}$ radicals.

Table S1 Crystallize size of e-BN, e-CN and e-BN/e-CN composites obtained from Scherer analysis.

\begin{tabular}{|l|l|}
\hline Photocatalysts & $\begin{array}{l}\text { Approximate Crystallize size } \\
\text { (D) }(\mathrm{nm}) \text { Scherer analysis }\end{array}$ \\
\hline e-BN & 12.5 \\
\hline e-CN & 6.5 \\
\hline $0.5 \mathrm{wt} \%$ e-BN/e-CN & 3.6 \\
\hline $1.0 \mathrm{wt} \%$ e-BN/e-CN & 2.7 \\
\hline $1.5 \mathrm{wt} \%$ e-BN/e-CN & 3.1 \\
\hline $2.0 \mathrm{wt} \%$ e-BN/e-CN & 3.2 \\
\hline
\end{tabular}

Table S2 Pseudo first-order kinetic data obtained for TCH degradation by e-CN and e-BN/e-CN composites. 


\begin{tabular}{|l|l|l|l|l|}
\hline Sl. No. & photocatalysts & $\mathrm{k}\left(\mathrm{min}^{-1}\right)$ & $\mathrm{R}^{2}$ & $\mathrm{t}_{1 / 2}$ \\
\hline 1 & e-CN & $12 \times 10^{-3}$ & 0.95101 & 53 \\
\hline 2 & $0.5 \mathrm{wt} \%$ e-BN/e-CN & $19 \times 10^{-3}$ & 0.95532 & 36 \\
\hline 3 & $1.0 \mathrm{wt} \%$ e-BN/e-CN & $23 \times 10^{-3}$ & 0.96583 & 28 \\
\hline 4 & $1.5 \mathrm{wt} \%$ e-BN/e-CN & $41 \times 10^{-3}$ & 0.99118 & 17 \\
\hline 5 & $2.0 \mathrm{wt} \%$ e-BN/e-CN & $31 \times 10^{-3}$ & 0.98586 & 22 \\
\hline
\end{tabular}

Table S3 Comparison study of various photocatalysts towards the photocatalytic TCH degradation and $\mathrm{H}_{2}$ evolution.

\begin{tabular}{|c|c|c|c|c|c|c|}
\hline \multicolumn{7}{|c|}{ Comparative study of photocatalytic TCH degradation } \\
\hline $\begin{array}{l}\text { Sl. } \\
\text { No. }\end{array}$ & Photocatalysts & $\begin{array}{l}\text { Synthesis } \\
\text { method }\end{array}$ & Light source & $\begin{array}{l}\text { Illumination } \\
\text { time (min) }\end{array}$ & $\begin{array}{c}\text { Degradation } \\
\%\end{array}$ & References \\
\hline 1 & BOI-CBBMO-3 & $\begin{array}{c}\text { In-situ } \\
\text { precipitation }\end{array}$ & Solar light & 60 & 87 & [1] \\
\hline 2 & HPW@UiO-66 & Solvothermal & Solar light & 60 & 87.24 & [2] \\
\hline 3 & $\mathrm{Ag}_{3} \mathrm{PO}_{4} / \mathrm{BiVO}_{4}$ & Precipitation & $\begin{array}{c}300 \mathrm{~W} \text { Xe } \\
\text { lamp }\end{array}$ & 180 & 91 & [3] \\
\hline 4 & $\mathrm{ZnIn}_{2} \mathrm{~S}_{4} / \mathrm{Ta}_{3} \mathrm{~N}_{5}$ & Hydrothermal & $\begin{array}{c}250 \mathrm{~W} X \mathrm{e} \\
\text { lamp }\end{array}$ & 180 & 89 & [4] \\
\hline 5 & $\mathrm{ZIF}-8 / \mathrm{MnFe}_{2} \mathrm{O}_{4}$ & Hydrothermal & $\begin{array}{c}300 \mathrm{~W} X e \\
\text { lamp }\end{array}$ & 90 & 83 & [5] \\
\hline 6 & e-BN/e-CN & Calcination & Solar light & 60 & 90 & This work \\
\hline \multicolumn{7}{|c|}{ Comparative study of photocatalytic $\mathrm{H}_{2}$ evolution } \\
\hline $\begin{array}{l}\text { Sl. } \\
\text { No. }\end{array}$ & Photocatalysts & $\begin{array}{l}\text { Synthesis } \\
\text { method }\end{array}$ & $\begin{array}{c}\text { Light } \\
\text { source }\end{array}$ & $\begin{array}{l}\text { Illumination } \\
\text { time (min) }\end{array}$ & $\begin{array}{c}\text { Rate of } \mathrm{H}_{2} \\
\text { evolution }\end{array}$ & References \\
\hline 7 & $\mathrm{ZnIn}_{2} \mathrm{~S}_{4} / \mathrm{Ta}_{3} \mathrm{~N}_{5}$ & Hydrothermal & $\begin{array}{c}300 \mathrm{~W} \text { xe } \\
\text { lamp }\end{array}$ & 300 & $\begin{array}{c}834.86 \\
\mu \mathrm{mol} \mathrm{g}^{-1} \mathrm{~h}^{-1}\end{array}$ & [4] \\
\hline 8 & $\mathrm{NiS}-\mathrm{WO}_{3} / \mathrm{g}-\mathrm{C}_{3} \mathrm{~N}_{4}$ & Solvothermal & 5 W LED & 300 & $\begin{array}{c}2929.1 \\
\mu \mathrm{molg}^{-1} \mathrm{~h}^{-1}\end{array}$ & [6] \\
\hline 9 & $\mathrm{BiPO}_{4} / \mathrm{S}-\mathrm{CN}$ & Calcination & $\begin{array}{c}500 \mathrm{~W} \text { xe } \\
\text { lamp }\end{array}$ & 180 & $11.7 \mu \mathrm{mol} / \mathrm{h}$ & [7] \\
\hline 10 & CdS/MXene & Solvothermal & $\begin{array}{c}300 \mathrm{~W} \times \mathrm{xe} \\
\text { lamp }\end{array}$ & 180 & $\begin{array}{c}2407 \\
\mu \mathrm{molg}^{-1} \mathrm{~h}^{-1}\end{array}$ & {$[8]$} \\
\hline
\end{tabular}




\begin{tabular}{|c|c|c|c|c|c|c|}
\hline 11 & $\mathrm{CoWO}_{4} / \mathrm{Mn}_{0.2} \mathrm{Cd}_{0.8} \mathrm{~S}$ & In-situ & $5 \mathrm{~W}$ LED & 300 & $408 \mu \mathrm{mol}$ & {$[9]$} \\
\hline 12 & $\mathrm{e}-\mathrm{BN} / \mathrm{e}-\mathrm{CN}$ & Calcination & $\begin{array}{c}125 \mathrm{~W} \mathrm{Xe} \\
\text { lamp }\end{array}$ & 60 & $\begin{array}{c}7359 \\
\mu \mathrm{molg}^{-1} \mathrm{~h}^{-1}\end{array}$ & This work \\
\hline
\end{tabular}

\section{References}

1. Kandi, D.; Behera, A.; Sahoo, S.; Parida, K. CdS QDs modified BiOI/Bi2MoO6 nanocomposite for degradation of quinolone and tetracycline types of antibiotics towards environmental remediation. Sep. Purif. Technol. 2020,253, p.117523-117558.

2. Subudhi, S.; Mansingh, S.; Swain, G.; Behera, A.; Rath, D.; Parida, K. HPW-anchored UiO-66 metal-organic framework: a promising photocatalyst effective toward tetracycline hydrochloride degradation and $\mathrm{H} 2$ evolution via Z-scheme charge dynamics. Inorg. Chem. 2019, 58, pp.4921-4934.

3. Gao, X.; Liang, C.; Gao, K.; Li, X.; Liu, J.; Li, Q. Z-scheme heterojunction Ag3PO4/BiVO4 with exposing high-active facets and stretching spatial charge separation ability for photocatalytic organic pollutants degradation. Appl. Surf. Sci. 2020, 524, p.146506-146536.

4. Xiao, Y.; Zhang, W.; Xing, Q.; Feng, X.; Jiang, Y.; Gao, Y.; Xu, H.; Zhang, J.; Ni, L.; Liu, Z. Eco-friendly synthesis of core/shell ZnIn2S4/Ta3N5 heterojunction for strengthened dual-functional photocatalytic performance. Int. J. Hydrog. Energy. 2020, 45, pp.30341-30356.

5. Wang, Z.; Lai, C.; Qin, L.; Fu, Y.; He, J.; Huang, D.; Li, B.; Zhang, M.; Liu, S.; Li, L.; Zhang, W. ZIF-8-modified MnFe2O4 with high crystallinity and superior photo-Fenton catalytic activity by Zn-O-Fe structure for TC degradation. Chem. Eng. J. 2020, 392, p. 124851-124863.

6. Zhang, L.; Hao, X.; Li, Y.; Jin, Z. Performance of WO3/g-C3N4 heterojunction composite boosting with NiS for photocatalytic hydrogen evolution. Appl. Surf. Sci. 2020, 499, p.143862-143885.

7. Long, D.; Chen, Z.; Rao, X.; Zhang, Y. Sulfur-Doped g-C3N4 and BiPO4 Nanorod Hybrid Architectures for Enhanced Photocatalytic Hydrogen Evolution under Visible Light Irradiation. ACS Appl. Energy Mater. 2020,3, pp.5024-5030.

8. Xiao, R.; Zhao, C.; Zou, Z.; Chen, Z.; Tian, L.; Xu, H.; Tang, H.; Liu, Q.; Lin, Z.; Yang, $X$. In situ fabrication of $1 \mathrm{D} \mathrm{CdS}$ nanorod/2D Ti3C2 MXene nanosheet Schottky heterojunction toward enhanced photocatalytic hydrogen evolution. Appl. Catal. B. 2020, 268,p.118382-118415.

9. Gong, H.; Wang, G.; Li, H.; Jin, Z.; Guo, Q. Mn0. 2Cd0. 8S nanorods assembled with 0D CoWO4 nanoparticles formed pn heterojunction for efficient photocatalytic hydrogen evolution. Int. J. Hydrog. Energy. 2020, 45, pp.26733-26745. 